ESAIM: PROCEEDINGS, September 2012, Vol. 37, p. 166-177

E. Cancès and S. Labbé, Editors

\title{
NUMERICAL STUDY OF ACOUSTIC MULTIPERFORATED PLATES
}

\author{
Abderrahmane Bendali ${ }^{1}$, M'Barek Fares ${ }^{2}$, Sophie Laurens ${ }^{3}$ and Sébastien \\ TORDEUX ${ }^{4}$
}

\begin{abstract}
It is rather classical to model multiperforated plates by approximate impedance boundary conditions. In this article we would like to compare an instance of such boundary conditions obtained through a matched asymptotic expansions technique to direct numerical computations based on a boundary element formulation in the case of linear acoustic.
\end{abstract}

\section{INTRODUCTION}

Multiperforated boundaries are involved in several industrial devices (liners [11], mufflers [2], combustion chambers [12], noise barriers ...). The occurrence of a high density of holes of small size has a significant impact on the noise generated by the devices.

Until recently, it was almost impossible to predict the effect of a multiperforated plate by a direct numerical computation. To overcome this bottleneck, many authors have proposed to replace the perforated wall by an effective impedance boundary condition of transmission type, see [16] for the case of linear acoustic. This approach is suitable for numerical computation since it requires no mesh refinement in the neighborhood of the holes. The rapid increase of numerical computational abilities allows for the validation of this method by means of high performance computing. In this article, we would like to address such an issue using the parallel boundary element library CESC of CERFACS.

In section 1, we describe the considered geometry and state the boundary-value problem obtained by assuming that the equations of linearized acoustics are valid everywhere. In section 2 , we briefly review some effective boundary conditions previously used for modeling multiperforated plates. Numerical experiments are presented in section 3 .

\footnotetext{
1 Toulouse University, INSA-Toulouse, Mathematical Institute of Toulouse (UMR- CNRS 5219), 135 avenue de Rangueil, F31077 Toulouse, France, and Electromagnetism and Acoustics, CERFACS, 42 Avenue Gaspard Coriolis, F-31100 Toulouse

2 Electromagnetism and Acoustics, CERFACS, 42 Avenue Gaspard Coriolis, F-31100 Toulouse, France

3 Toulouse University, INSA-Toulouse, Mathematical Institute of Toulouse (UMR- CNRS 5219), 135 avenue de Rangueil, F31077 Toulouse, France

${ }^{4}$ Projet Magique 3D, INRIA Bordeaux Sud-Ouest and LMA - UMR CNRS 5142, Université de Pau et des Pays de l'Adour, France
}

(C) EDP Sciences, SMAI 2012 


\section{Statement of the Problem}

\subsection{Description of the propagation domain}

We consider an infinite waveguide $T$ with rectangular section

$$
\mathbf{T}=\left\{\mathbf{x} \in \mathbb{R}^{3}: \mathbf{x}_{1} \in\right] 0, h_{1}\left[\text { and } \mathbf{x}_{2} \in\right] 0, h_{2}[\} .
$$

This domain is split into two parts by a multiperforated plate with zero thickness

$$
\mathrm{P}=\left\{\mathbf{x} \in \mathbb{R}^{3}: \mathbf{x}_{1} \in\right] 0, h_{1}\left[, \mathbf{x}_{2} \in\right] 0, h_{2}\left[\text { and } \mathbf{x}_{3}=0\right\} .
$$

The wall $\mathrm{P}$ is perforated by a grating of characteristic lengths $\eta_{1}$ and $\eta_{2}$ of holes $\Theta^{m}$ of radius $\delta>0$

$$
\left\{\begin{array}{l}
\mathbf{x}^{m}=\left(\frac{\eta_{1}}{2}, \frac{\eta_{2}}{2}, 0\right)+\left(\left(m_{1}+m_{2} \alpha\right) \eta_{1}, m_{2} \eta_{2}, 0\right) \text { with } \alpha \in[0,1[ \\
\Theta^{m}=\left\{\mathbf{x} \in \mathbb{R}^{3}:\left\|\mathbf{x}-\mathbf{x}^{m}\right\|<\delta \text { and } \mathbf{x}_{3}=0\right\} \\
\text { with } m \in \mathbb{S}=\left\{\left(m_{1}, m_{2}\right) \in \mathbb{Z}^{2}: \Theta^{m} \subset \mathrm{P}\right\}
\end{array}\right.
$$

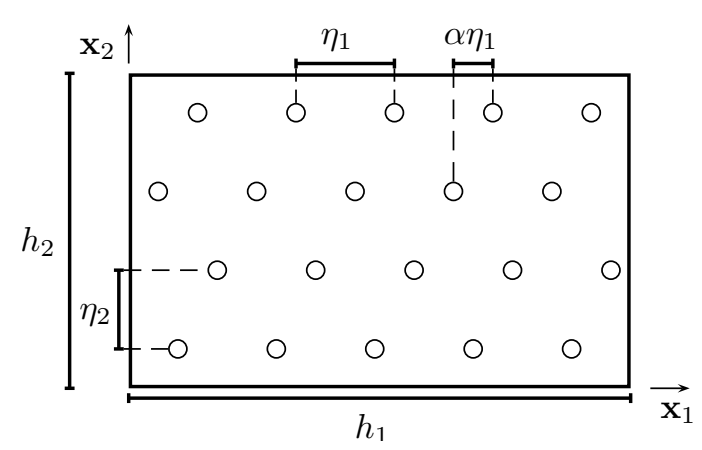

FIGURE 1. The multiperforated plate

The spacing between two holes is large enough so that short range interactions can be neglected, i.e., $\delta / \min \left(\eta_{1}, \eta_{2}\right)=o(1)$. We can thus define the propagation domain:

$$
\Omega=\mathrm{T} \backslash \mathrm{P}_{\text {perf }} \text { with } \mathrm{P}_{\text {perf }}=\mathrm{P} \backslash\left(\bigcup_{m \in \mathbb{S}} \Theta^{m}\right) .
$$

\subsection{Linear acoustics modeling}

The time variable and the speed of sound are respectively denoted by $t$ and $c$. Let us recall that in the context of linear acoustic the pressure field $p: \Omega \times \mathbb{R}_{+} \mapsto \mathbb{R}$ is governed by a scalar wave equation

$$
\frac{\partial^{2} p}{\partial t^{2}}(\mathbf{x}, t)-c^{2} \Delta p(\mathbf{x}, t)=0 \quad \text { in } \Omega .
$$

The disturbed velocity vector field $\mathbf{v}: \Omega \times \mathbb{R}_{+} \mapsto \mathbb{R}^{3}$ is linked to the pressure by linearized Euler's equations

$$
\rho_{0} \frac{\partial \mathbf{v}}{\partial t}(\mathbf{x}, t)+\nabla p(\mathbf{x}, t)=0 \quad \text { in } \Omega
$$


where $\rho_{0}$ is the density of the fluid at rest. The boundary of the physical domain is supposed to be a perfectly rigid wall

$$
\mathbf{v} \cdot \mathbf{n}=0 \text { on } \partial \Omega=\partial T \backslash\left(\mathrm{P} \backslash\left(\bigcup_{m \in \mathbb{S}} \Theta^{m}\right)\right),
$$

with $\mathbf{n}$ the normal unit vector outwardly directed to $\Omega$. Considering a wave with pulsation $\omega$ and wave number $k=\omega / c$, we denote also by $p$ and $\mathbf{v}$ the phasors of respectively the pressure and the velocity

$$
p(\mathbf{x}, t)=\Re(p(\mathbf{x}) \exp (-i \omega t)) \quad \text { and } \quad \mathbf{v}(\mathbf{x}, t)=\Re(\mathbf{v}(\mathbf{x}) \exp (-i \omega t)) .
$$

In the sequel we consider the well-posed boundary-value problem

$$
\left\{\begin{array}{l}
\text { Find } p: \Omega \longrightarrow \mathbb{C} \text { satisfying } \\
\chi p \in H^{1}(\Omega) \text { for all } \chi \in \mathcal{D}\left(\mathbb{R}^{3}\right) \\
\Delta p(\mathbf{x})+k^{2} p(\mathbf{x})=0 \quad \text { in } \Omega \\
\frac{\partial p}{\partial n}(\mathbf{x})=0 \quad \text { on } \partial \Omega \\
p(\mathbf{x})-\exp \left(i k \mathbf{x}_{3}\right) \text { is outgoing, see [4] or [10] for example }
\end{array}\right.
$$

which provides a model for the time-harmonic waves standing in this acoustic system when it is fed by the propagating mode coming from $\mathbf{x}_{3}=-\infty$.

Remark 1. For a waveguide with small height and width $\left(k h_{1}<\pi\right.$ and $\left.k h_{2}<\pi\right)$ the harmonic pressure $p$ can be expressed as

$$
\begin{cases}p(\mathbf{x}, t)=\exp \left(i k \mathbf{x}_{3}\right)+R \exp \left(-i k \mathbf{x}_{3}\right)+w_{+}(\mathbf{x}) & \text { for } \mathbf{x}_{3}<0, \\ p(\mathbf{x}, t)=T \exp \left(i k \mathbf{x}_{3}\right)+w_{-}(\mathbf{x}) & \text { for } \mathbf{x}_{3}>0 .\end{cases}
$$

Parameters $R$ and $T$ are respectively the reflection and transmission coefficients. Both functions $w_{-}$and $w_{+}$ are evanescent in the $\mathbf{x}_{3}$ direction (exponential decay):

$$
w_{-}(\mathbf{x})=\underset{\mathbf{x}_{3} \rightarrow-\infty}{o}(1) \quad \text { and } \quad w_{+}(\mathbf{x})=\underset{\mathbf{x}_{3} \rightarrow+\infty}{o}(1)
$$

\section{The APPRoximate TRANSMission MODEL FOR MUlTiPERFORATED PLATES}

For a large number of holes, even when resorting to high performance computing, the direct numerical solution of problem (9) is generally out of reach. To overcome this difficulty a solution consists in modeling the multiperforated plate by an equivalent transmission condition (also called impedance condition) which requires no mesh refinement.

These approximate conditions have been well-studied by the acoustic community both from the theoretical and experimental point of view. It can either be achieved using heuristic approaches based on a physical reasonning [16] or by means of asymptotic analysis, see [1], [2] or [3] and references therein. The last approach benefits from a rigorous framework based on the matching of asymptotic expansions techniques [9].

The conclusion of these studies can be physically interpreted as follows: the multiperforated plate can be modeled as an impenetrable moving membrane of surface mass $\rho_{S}$. This leads to a system of partial differential equations posed on the domain

$$
\widetilde{\Omega}=\mathrm{T} \backslash \mathrm{P}
$$


governing the pressure field of the fluid $\widetilde{p}$, the flow velocity vector field of the fluid $\widetilde{\mathbf{v}}$ and the surface velocity of the membrane $\widetilde{\mathbf{V}}=\left(\widetilde{\mathbf{V}}_{1}, 0,0\right)$

$$
\left\{\begin{array}{l}
\text { Acoustic equations in the fluid: } \\
\frac{\partial^{2} \widetilde{p}}{\partial t^{2}}(\mathbf{x}, t)-c^{2} \Delta \widetilde{p}(\mathbf{x}, t)=0 \text { in } \widetilde{\Omega} \\
\rho_{0} \frac{\partial \widetilde{\mathbf{v}}}{\partial t}(\mathbf{x}, t)+\nabla \widetilde{p}(\mathbf{x}, t)=0 \quad \text { in } \widetilde{\Omega} \\
\widetilde{\mathbf{v}}(\mathbf{x}, t) \cdot \mathbf{n}=0 \quad \text { on } \partial T . \\
\text { Impenetrability of the membrane: } \\
\widetilde{\mathbf{v}}_{1}\left(\mathbf{x}_{1}, \mathbf{x}_{2}, 0_{-}, t\right)=\widetilde{\mathbf{v}}_{1}\left(\mathbf{x}_{1}, \mathbf{x}_{2}, 0_{+}, t\right)=\widetilde{\mathbf{V}}_{1}\left(\mathbf{x}_{1}, \mathbf{x}_{2}, t\right) \\
\text { Second Newton's law for the membrane: } \\
\rho_{S} \frac{\partial \widetilde{\mathbf{V}}_{1}}{\partial t}\left(\mathbf{x}_{1}, \mathbf{x}_{2}, t\right)=\widetilde{p}\left(\mathbf{x}_{1}, \mathbf{x}_{2}, 0_{-}, t\right)-\widetilde{p}\left(\mathbf{x}_{1}, \mathbf{x}_{2}, 0_{+}, t\right) .
\end{array}\right.
$$

In the case of an incident harmonic wave coming from $\mathbf{x}_{3}=-\infty$, this system simplifies into a problem involving only $\widetilde{p}: \widetilde{\Omega} \longrightarrow \mathbb{C}$

$$
\left\{\begin{array}{l}
\chi \widetilde{p} \in H^{1}(\widetilde{\Omega}) \text { for all } \chi \in \mathcal{D}\left(\mathbb{R}^{3}\right) \\
\Delta \widetilde{p}(\mathbf{x})+k^{2} \widetilde{p}(\mathbf{x})=0 \quad \text { in } \widetilde{\Omega} \\
\frac{\partial \widetilde{p}}{\partial n}(\mathbf{x})=0 \quad \text { on } \partial \mathrm{T}, \\
\frac{\partial \widetilde{p}}{\partial \mathbf{x}_{1}}\left(\mathbf{x}_{1}, \mathbf{x}_{2}, 0_{-} \text {or } 0_{+}\right)=\frac{K_{R}}{\mathrm{~A}}\left(\widetilde{p}\left(\mathbf{x}_{1}, \mathbf{x}_{2}, 0_{+}\right)-\widetilde{p}\left(\mathbf{x}_{1}, \mathbf{x}_{2}, 0_{-}\right)\right) \\
\widetilde{p}(\mathbf{x})-\exp \left(i k \mathbf{x}_{3}\right) \text { is outgoing }
\end{array}\right.
$$

expressed in terms of the so-called Rayleigh conductivity $K_{R}$ of the hole and the surface of an unit-cell of the network $A=\eta_{1} \eta_{2}$. These quantities are related to the surface mass of the membrane by:

$$
\rho_{S}=\frac{\rho_{0} \mathrm{~A}}{K_{R}}
$$

Remark 2 (Physical interpretation of the transmission condition). In the theory of acoustic, it is well known that the effect of a small perforation in a rigid plate can be modeled by two acoustic point sources located on each side of the plate, see [16] for example. The algebraic fluxes of fluid coming from these sources $\Phi_{-}$and $\Phi_{+}$ are opposite (due to the conservation of mass) and related to the difference of pressure on the two sides of the plate $\widetilde{p}_{+}-\widetilde{p}_{-}$by an analogous relation to the second Newton's law:

$$
\rho_{0} \frac{d \Phi_{-}}{d t}=-\rho_{0} \frac{d \Phi_{+}}{d t}=K_{R}\left(\widetilde{p}_{+}-\widetilde{p}_{-}\right) .
$$

The multiplicative constant $K_{R}$ is traditionally called the Rayleigh conductivity, see [13]. It is given by

$$
K_{R}=2 \delta
$$

in the case of linear acoustics for a circular hole of radius $\delta$ in a plate of negligible thickness.

A fluid crossing a grating of holes has a complex structure in the neighborhood of the perforations but can be seen as a homogeneous fluid at large scale. The velocity of fluid is then determined by distributing over one unit 
cell of the network the flux coming from one hole:

$$
\widetilde{\mathbf{v}}_{3}\left(\mathbf{x}_{1}, \mathbf{x}_{2}, 0_{-}, t\right)=-\frac{\Phi_{-}}{\mathrm{A}} \quad \text { and } \quad \widetilde{\mathbf{v}}_{3}\left(\mathbf{x}_{1}, \mathbf{x}_{2}, 0_{+}, t\right)=\frac{\Phi_{+}}{\mathrm{A}}
$$

This leads to the transmission conditions

$$
\left\{\begin{array}{l}
\widetilde{\mathbf{v}}_{1}\left(\mathbf{x}_{1}, \mathbf{x}_{2}, 0_{-}, t\right)=\widetilde{\mathbf{v}}_{1}\left(\mathbf{x}_{1}, \mathbf{x}_{2}, 0_{+}, t\right) \\
\mathrm{A} \frac{\partial \widetilde{\mathbf{v}}_{1}}{\partial t}\left(\mathbf{x}_{1}, \mathbf{x}_{2}, 0_{-}, t\right)=K_{R}\left(\widetilde{p}\left(\mathbf{x}_{1}, \mathbf{x}_{2}, 0_{-}, t\right)-\widetilde{p}\left(\mathbf{x}_{1}, \mathbf{x}_{2}, 0_{+}, t\right)\right) \\
\mathrm{A} \frac{\partial \widetilde{\mathbf{v}}_{1}}{\partial t}\left(\mathbf{x}_{1}, \mathbf{x}_{2}, 0_{+}, t\right)=K_{R}\left(\widetilde{p}\left(\mathbf{x}_{1}, \mathbf{x}_{2}, 0_{-}, t\right)-\widetilde{p}\left(\mathbf{x}_{1}, \mathbf{x}_{2}, 0_{+}, t\right)\right)
\end{array}\right.
$$

Remark 3. The solution of the approximate model can be analytically computed. Its solution depends only on the $\mathbf{x}_{3}$ coordinate and is given by

$$
\begin{cases}\widetilde{p}_{-}(\mathbf{x})=\exp \left(i k \mathbf{x}_{3}\right)+\widetilde{R} \exp \left(-i k \mathbf{x}_{3}\right) & \text { for } \mathbf{x}_{3}<0 \\ \widetilde{p}_{+}(\mathbf{x})=\widetilde{T} \exp \left(i k \mathbf{x}_{3}\right) & \text { for } \mathbf{x}_{3}>0\end{cases}
$$

Inserting (20) in the transmission conditions (19) leads to the reflection and transmission coefficients:

$$
\widetilde{R}=\frac{-\frac{i k \mathrm{~A}}{K_{R}}}{2-\frac{i k \mathrm{~A}}{K_{R}}} \quad \text { and } \quad \widetilde{T}=\frac{2}{2-\frac{i k \mathrm{~A}}{K_{R}}}
$$

\section{A NUMERICAL EXPERIMENT}

We consider two multiperforated plates with almost the same geometrical characteristic lengths

$$
h_{1}=5 \mathrm{~cm}, \quad h_{2}=5 \mathrm{~cm}, \quad \eta_{1}=5 \mathrm{~mm}, \quad \eta_{2}=5 \mathrm{~mm}, \quad \delta=0.225 \mathrm{~mm} .
$$

In the first configuration, the grating is straight, $\alpha=0$ (see (3) and Figure 1 for the definition of $\alpha$ ) and tilted in the second one, $\alpha=1 / 2$, see Figure 2 . 


\begin{tabular}{llllllllll|}
0 & 0 & 0 & 0 & 0 & 0 & 0 & 0 & 0 & 0 \\
0 & 0 & 0 & 0 & 0 & 0 & 0 & 0 & 0 & 0 \\
0 & 0 & 0 & 0 & 0 & 0 & 0 & 0 & 0 & 0 \\
0 & 0 & 0 & 0 & 0 & 0 & 0 & 0 & 0 & 0 \\
0 & 0 & 0 & 0 & 0 & 0 & 0 & 0 & 0 & 0 \\
0 & 0 & 0 & 0 & 0 & 0 & 0 & 0 & 0 & 0 \\
0 & 0 & 0 & 0 & 0 & 0 & 0 & 0 & 0 & 0 \\
0 & 0 & 0 & 0 & 0 & 0 & 0 & 0 & 0 & 0 \\
0 & 0 & 0 & 0 & 0 & 0 & 0 & 0 & 0 & 0 \\
0 & 0 & 0 & 0 & 0 & 0 & 0 & 0 & 0 & 0 \\
\hline
\end{tabular}

Figure 2. Multiperforated plates with $\alpha=0$ and $\alpha=\frac{1}{2}$

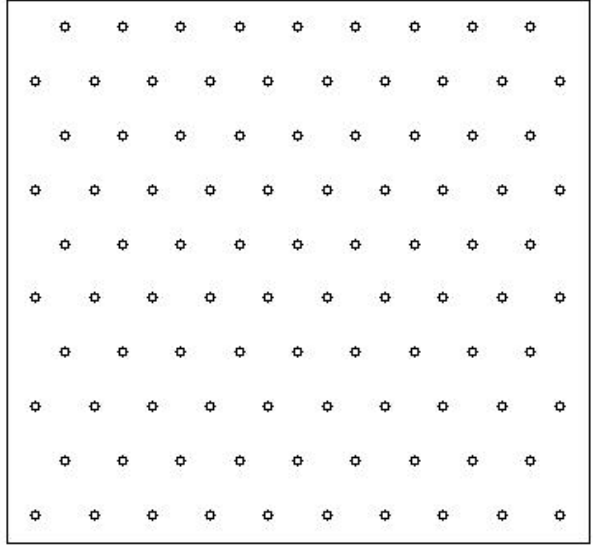

The meshes, see Figures 3, were generated with the proprietary licensed software Altair HyperMesh library (http://www.hyperworks.fr) and its scripting language to ensure a local refinement in the neighborhood of each hole, see Figure 4.
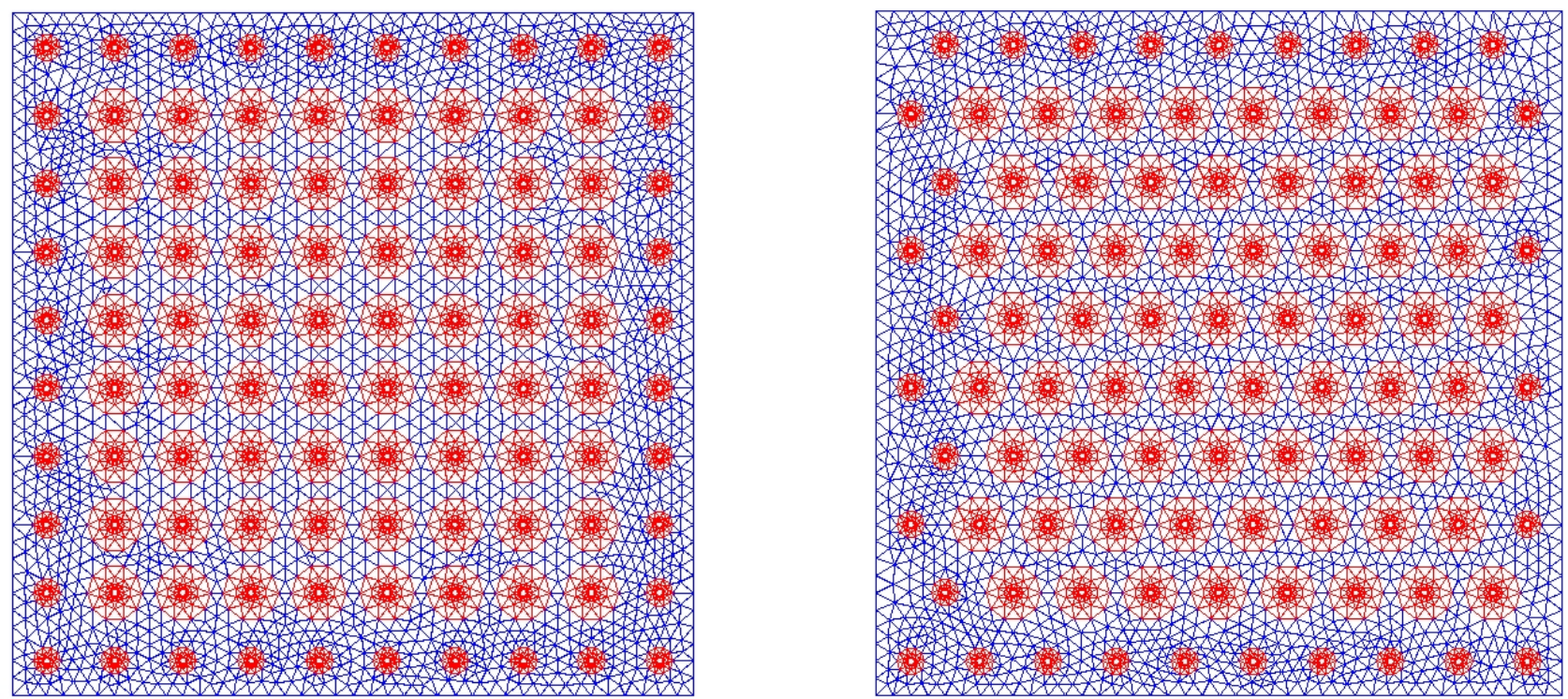

FIgURE 3. The meshes of the multiperforated plates for $\alpha=0$ and $\alpha=1 / 2$ 


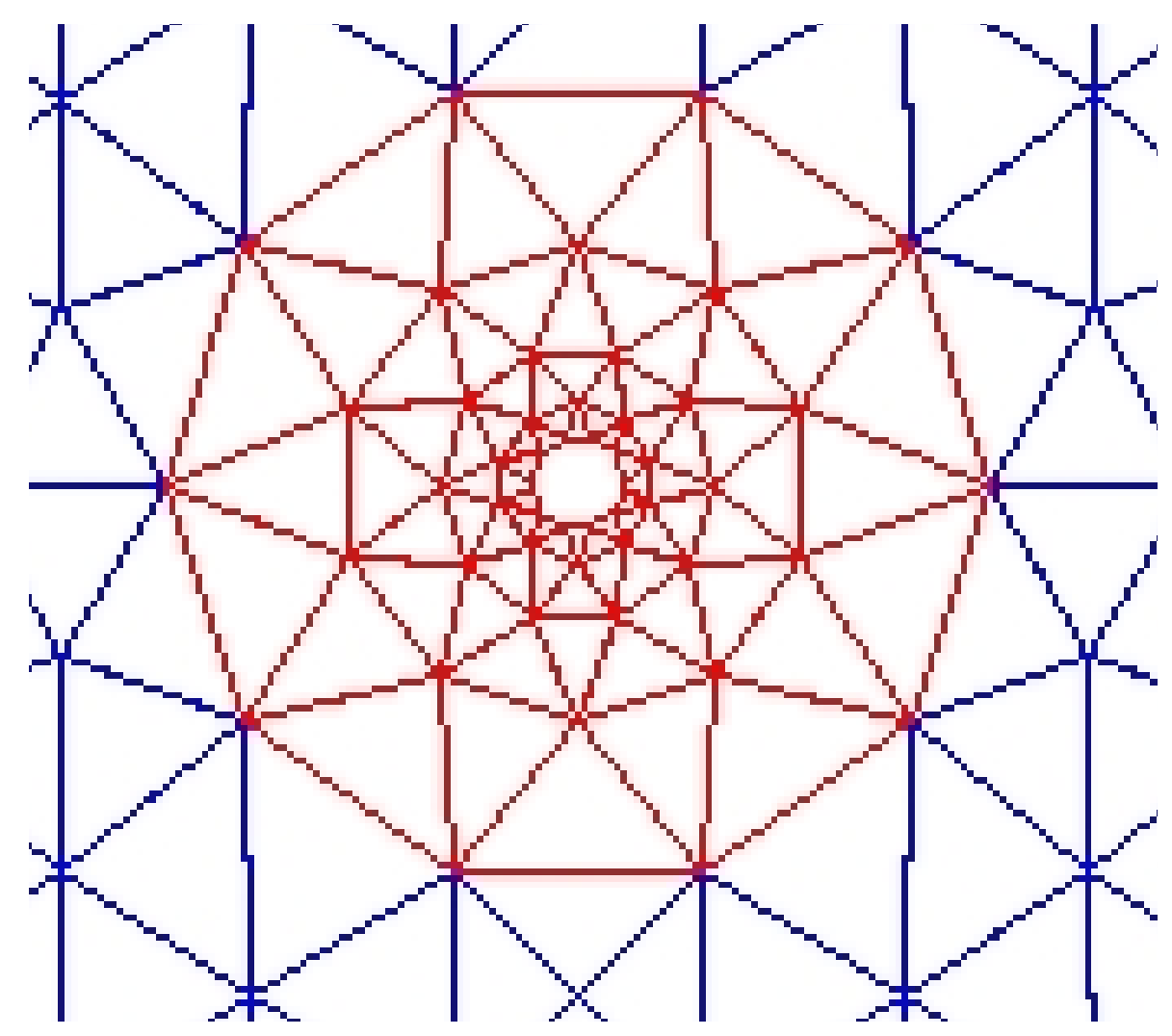

Figure 4. The local refinement Patch

The outgoing condition is approximated by truncating the domain at $h_{3}=2 \mathrm{~cm}$ from the multiperforated plate, see Figure 5. On the two sections $\mathbf{x}_{3}=-h_{3}$ and $\mathbf{x}_{3}=h_{3}$ is prescribed the following accurate absorbing boundary conditions :

$$
\left\{\begin{array}{l}
\frac{\partial p}{\partial \mathbf{x}_{1}}\left(\mathbf{x}_{1}, \mathbf{x}_{2},-h_{3}\right)+i k p\left(\mathbf{x}_{1}, \mathbf{x}_{2},-h_{3}\right)=2 i k \exp \left(-i k h_{3}\right) \\
\frac{\partial p}{\partial \mathbf{x}_{1}}\left(\mathbf{x}_{1}, \mathbf{x}_{2}, h_{3}\right)-i k p\left(\mathbf{x}_{1}, \mathbf{x}_{2}, h_{3}\right)=0
\end{array}\right.
$$

These conditions are rather accurate since they are obtained by neglecting the evanescent modes which are exponentially decaying. 


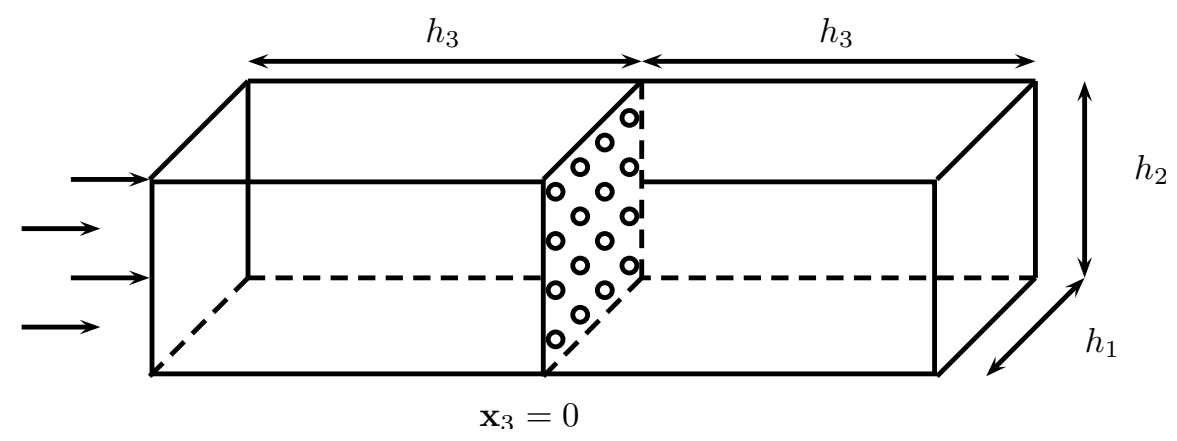

FiguRE 5. The truncated domain of computation

The numerical experiments have been achieved with the boundary element library CESC. We have chosen to use a double layer formulation [15] to discretize the system of equations (9) and numerically compute its reflection coefficient $R$. In order to check the convergence of the method, we have compared the numerical results with approximately $4 \times 10^{4}$ degrees of freedom (d.o.f.) to the results with $1.6 \times 10^{5}$ d.o.f. Both numerical approximations of the reflection coefficients coincide up to the third digit.

We have considered 26 frequencies (from $500 \mathrm{~Hz}$ to $3000 \mathrm{~Hz}$ with a $100 \mathrm{~Hz}$ step). The determination of the transmission and reflection coefficients at each frequency requires to form and invert a linear system with a dense matrix of approximately 40,000 unknowns. It was carried on on a parallel platform having 400 processors. It required a rather large computational time $\left(5.2 \times 10^{6} \mathrm{~s}\right.$ i.e. $\left.1.3 \times 10^{4} \mathrm{~s} \cdot \mathrm{cpu} \mathrm{u}^{-1}\right)$.

The modulus $|R|$ and the phase angle of the reflection coefficients respectively obtained from the direct numerical solution and the homogenized approximate model are depicted in Figure 6 for the straight case $(\alpha=0)$ and in Figure 7 for the tilted case $(\alpha=1 / 2)$.

The conclusions that can be drawn from these plots are the following:

- As predicted by the theory, there is almost no difference between the straight case and the tilted case.

- Even if the overall behavior of the reflection coefficient is well predicted, an error of $10 \%$ on its modulus and of $5 \%$ on its phase angle can be observed. 

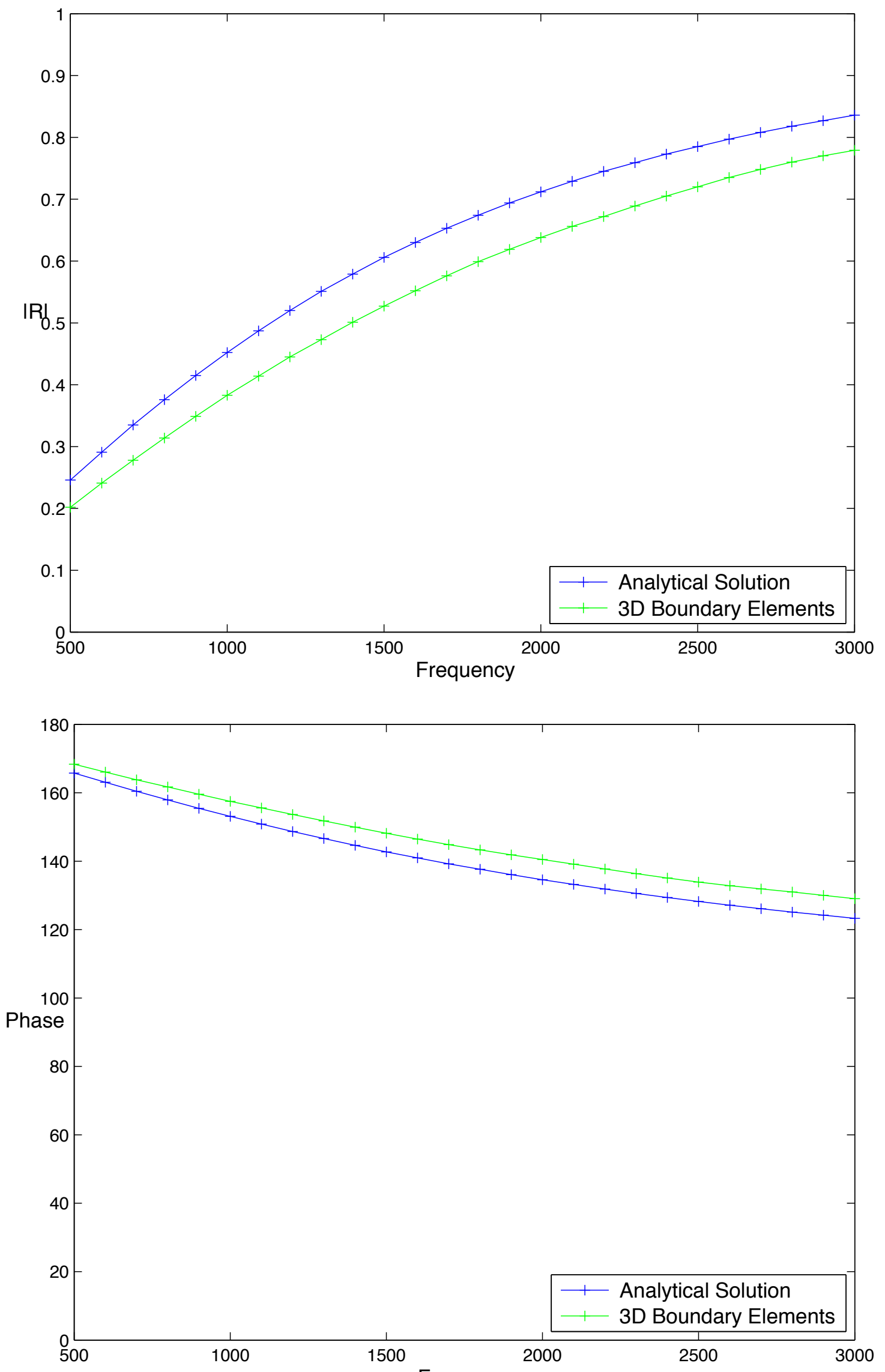
ESAIM: PROCEEDINGS
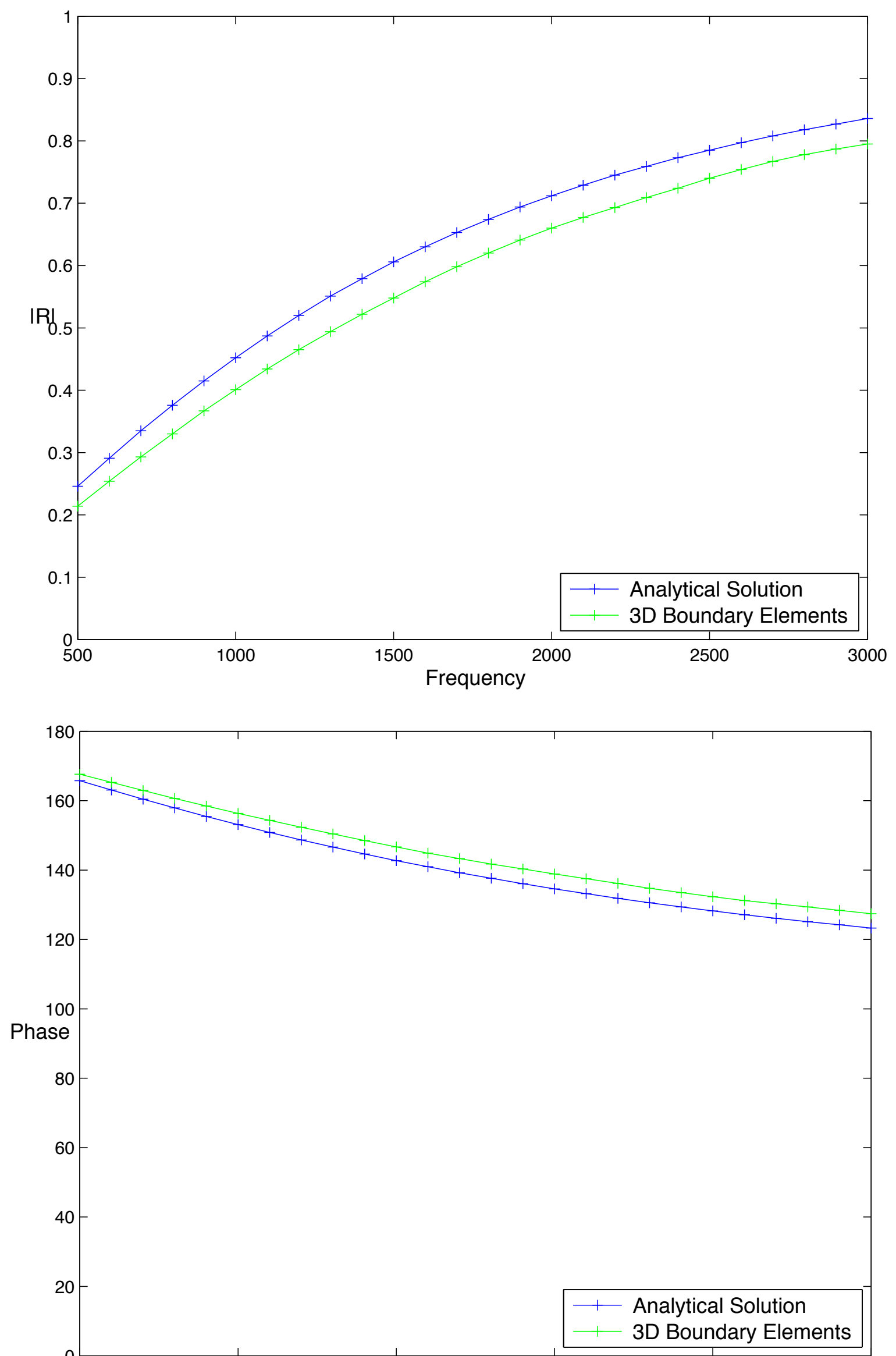


\section{Conclusion}

A numerical validation of the approximate transmission condition modeling the reflection and the transmission of acoustic waves by a multiperforated plate has been carried out. The modulus and the phase angle for the reflection coefficients obtained through a direct numerical solution and the use of the homogenized transmission condition have exactly the same overall behavior even if a discrepancy between the two results gives rise to an error of about $10 \%$. To reduce this discrepancy, it would be interesting in our opinion to carry out further investigations.

First, the homogenized transmission conditions can be improved by taking into account the short rang interactions. These objectives can be achieved by performing a multi-scale in place of a two-scale asymptotic analysis.

Moreover, in most applications (combustion chamber, liners), linear acoustics is not generally used for modeling the behavior of the acoustic field in the proximity of the holes. Reproducing this approach for these more complicated models seem to be rather challenging. One can refer to [14] for viscosity and to [6-8] for aeroacoustic effect.

The last issue is the choice of the numerical method. We have chosen to use a direct solution by a plain boundary element method without accelerating it by a Fast Multipole Method (FMM) (see, e.g., [5]). This choice is motivated by the fact that the problem being considered here is in the low frequencies range and at the best of authors knowledge no completely satisfactory FMM is available in this case.

\section{ACKNOWLEDGMENTS.}

This work was supported by the French National Research Agency under grant no. ANR-08-SYSC-001.

\section{REFERENCES}

[1] A. S. Bonnet-BenDhia, D. Drissi, and N. Gmati. Simulation of muffler's transmission losses by a homogenized finite element method. J. Comput. Acoust., 12(3):447-474, 2004.

[2] A. S. Bonnet-BenDhia, D. Drissi, and N. Gmati. Mathematical analysis of the acoustic diffraction by a muffler containing perforated ducts. Math. Models Methods Appl. Sci., 15(7):1059-1090, 2005.

[3] G. A. Chechkin and R. R. Gadyl'shin. On boundary-value problems for the Laplacian in bounded and in unbounded domains with perforated boundaries. J. Differential Equations, 216(2):502-522, 2005.

[4] C. I. Goldstein. A finite element method for solving Helmholtz type equations in waveguides and other unbounded domains. Math. Comp., 39(160):309-324, 1982.

[5] N. A. Gumerov and R. Duraiswamy. Fast Multipole Methods for the Helmholtz Eqaution in Three Dimensions. Elsevier, Amsterdam, 2004.

[6] M. S. Howe. On the theory of unsteady high reynolds number flow through a circular aperture. Proc. R. Soc. Lond. A, 336(1725):205-223, june 1979.

[7] M. S. Howe. Acoustics of fluid-structure interactions. Cambridge Monographs on Mechanics. Cambridge University Press, Cambridge, 1998.

[8] I. J. Hughes and A. P. Dowling. The absorption of sound by perforated linings. Journal of Fluid Mechanics, 218(-1):299-335, 1990.

[9] A. M. Il'in. Matching of asymptotic expansions of solutions of boundary value problems, volume 102 of Translations of Mathematical Monographs. American Mathematical Society, Providence, RI, 1992.

[10] M. Lenoir and A. Tounsi. The localized finite element method and its application to the two-dimensional sea-keeping problem. SIAM J. Numer. Anal., 25(4):729-752, 1988.

[11] S. Lidoine, I. Terrasse, T. Abboud, and A. Bennani. Numerical prediction of SDOF-Perforated Plate Acoustic Treatment Impedance. Part 1: Linear domain. In 13th AIAA/CEAS Aeroacoustics Conference(28th AIAA Aeroacoustics Conference). American Institute of Aeronautics and Astronautics, 2007.

[12] S. Mendez and J. D. Eldredge. Acoustic modeling of perforated plates with bias flow for Large-Eddy Simulations. Journal of Computational Physics, 228(13):4757-4772, 2009.

[13] B. J. W. S. Rayleigh. The theory of sound. Dover publications, 1945. 
[14] J. Sanchez-Hubert and E. Sanchez-Palencia. Acoustic fluid flow through holes and permeability of perforated walls. Journal of Mathematical Analysis and Applications, 87(2):427 - 453, 1982.

[15] S.A. Sauter and C. Schwab. Boundary element methods, volume 39 of Springer Series in Computational Mathematics. SpringerVerlag, Berlin, 2011. Translated and expanded from the 2004 German original.

[16] E. O. Tuck. Matching problems involving flow through small holes. Advances in Applied Mechanics, 15:89 - 158, 1975. 